\title{
Technology Considerations for Inclusion of Survivability in MDAO
}

\author{
Natalia M. Alexandrov ${ }^{1}$ \\ NASA Langley Research Center, Hampton VA 23681-2199
}

\begin{abstract}
Rising traffic density, along with autonomy and diversity of vehicles in the air, will fundamentally change the safety environment of the future air transportation system. The change in risk is two-fold: increasing chances of mid-air collisions with non-cooperative objects and increasing chances of crashes over highly populated areas. The changing nature of the vehicles populating the airspace means that civilian aircraft design must now explicitly include considerations of survivability in the event of collision with other vehicles, as well as prevention of damage to people, animals and property on the ground, to a much greater extent than today. This paper offers a preliminary perspective on how MDAO could contribute toward these goals. One of the conclusions is that, in contrast to traditional vehicle design, to accommodate the complexity of the future airspace safely and efficiently, vehicle design requirements, modeling, and design optimization must be closely connected to the properties of the airspace, including those of other vehicles in the air. Thus, the total measure of a vehicle's survivability should include the traditional survivability in malfunction scenarios, combined with new considerations of survivability in collisions and survivability of the public on the ground.
\end{abstract}

\section{Introduction}

$\mathrm{T}$ he changing nature of airspace presents vast challenges to vehicle design and airspace control. Whether a massive influx of on-demand urban air transportation, such as air taxis ${ }^{2}$, is imminent, realistic or desirable is open to argument. However, the density of air traffic over highly populated areas will increase, at the very least with the growing presence of unmanned aerial systems (UAS), including autonomous (self-governing) vehicles. Rising densities, along with autonomy and diversity of vehicles in the air, will fundamentally change the safety environment of the future air transportation system. On the one hand, increased automation and autonomy present an opportunity to manage greater densities of traffic, not amenable to handling by humans alone. On the other, machine-learning-based autonomous mechanisms are subject to a great deal of uncertainty in realistic environments, given that traditional validation and verification techniques no longer apply.

The Federal Aviation Administration (FAA) is partnering with industry to promote safe use of $\mathrm{UAS}^{3}$. The main approach to safety is separation of various domains in the air and on the ground, e.g., via Geofencing ${ }^{4}$. Separation and other risk mitigation measures have been proposed in, e.g., [Ref. 1]. These measures will safeguard operations in large portions of airspace. However, they rely on voluntary cooperation of all participants and a minimum level of functioning equipage. While the majority of the airspace participants will exhibit cooperative behavior, some will not. UAS interfering with firefighting efforts in California in July 2015 are one well-known example of noncooperative behavior with potentially serious consequences ${ }^{5}$. Non-cooperative behavior, whether due to inadequate equipment, lack of information, or ill intent, will be aggravated with increasing air traffic density.

Civilian UAS are still relatively small, light, short-range, and low-altitude vehicles, vulnerable to bad weather. However, the hard components of UAS - the battery, the engine, the camera - present danger to engines on large aircraft and to windshields on small aircraft. Recent computational investigations into UAS ingestion into engines

\footnotetext{
${ }^{1}$ Senior Research Scientist, Mail Stop 442, AIAA Associate Fellow

${ }^{2} \mathrm{http} / / / \mathrm{www} \cdot$ businessinsider.com/uber-elevate-flying-cars-2016-10

${ }^{3} \mathrm{http} / / /$ knowbeforeyoufly.org/

${ }^{4} \mathrm{http}: / / \mathrm{www}$.gizmag.com/nasa-air-traffic-control-drones/36509/

${ }^{5}$ http://www.npr.org/2015/07/23/425654435/california-firefighters-forced-to-call-off-missions-after-droneinterference
} 
confirm the potential hazardous effects of collision with UAS [Refs 2, 3]. Non-cooperative behavior, whether unwitting or intentional, will present growing danger to aircraft carrying passengers, as well as humans on the ground, as availability of UAS and density of UAS traffic increase.

If the vision of on-demand urban transportation materializes, the density of larger-than-drones traffic over highly populated areas will present a serious safety challenge as well. The safety record of general aviation (and general aviation would subsume urban air transportation) is considerably worse than that of commercial aviation ${ }^{6}$. Piloted air taxis will be subject to the same problems as piloted GA aircraft; and arguably, fully autonomous aircraft will remain impractical for some time.

To summarize, the new risks are two-fold: increasing chances of mid-air collisions with non-cooperative objects and increasing chances of crashes over highly populated areas. This suggests two major directions for mitigating the risks: maintain flight or, if you cannot, complete flight so as to avoid or minimize damage on the ground; and in all cases, minimize the loss of life. While a great deal of mitigation would have to rely on the developments in sensors and adaptive learning algorithms, vehicle design would have an important role to play as well; hence the need to consider potential contributions from MDAO. In the following sections, we offer an initial analysis of the survivability problem, as a function of technology, and comment on the role of MDAO in reducing the new risks.

\section{Survivability in Collisions}

Traditionally, survivability has been a concern of the military domain. Civilian aircraft design has had to deal with a more limited set of survivability issues, such as the impact of bird strikes, weather, fire, and mechanical failure. Danger from collisions with other aircraft has been prevented through tight regulation and control of the airspace, with predetermined separation constraints; and directions provided by the external air traffic control system and some on-board equipment. Increasing density of traffic and the potential presence of non-cooperative vehicles mean that civilian aircraft design must now explicitly include considerations of survivability in the event of collision with other vehicles.

One of the most important features of mid-air encounters with non-cooperative objects is the extremely short duration of the event. For instance, during the "Miracle-on-the-Hudson" incident (15 January 2009, US Airways Flight 1549), only 1.5 seconds elapsed between the pilots noticing the flock of birds and the bird strikes that ultimately downed the aircraft.

The external air traffic control system, the human pilots, and (at present) the aircraft control system cannot take protective action at such time scales. Maneuverability of large passenger aircraft and the effects of sharp maneuvers on passengers are also a factor. To ensure the safety of humans on an aircraft, intelligent systems must detect (sensing and perception are involved) a non-cooperative UAS automatically, make an autonomous machine decision, and affect the controls to implement it, all in time for the aircraft systems' actions to weaken the collision and mitigate its consequences. Arguably, these technologies, while in development, will not be mature for quite some time. Perception and real-time autonomous machine decision-making are particularly difficult problems, even assuming adequate sensors.

The premise of this discussion is that the risk of collision of aircraft carrying humans (pilots and/or passengers) with non-cooperative aircraft will increase, despite automatic collision avoidance systems, such as Traffic Alert and Collision Avoidance System (TCAS) [Ref. 4] and its probable successor, the Airborne Collision Avoidance System (ACAS X) [Ref. 5]. Thus, the discipline of survivability has to be included in Multidisciplinary Design Analysis and Optimization (MDAO), on par with traditional disciplines or in some other appropriate form.

\section{A. Summary of a Preliminary Study}

In a previous paper [Ref. 6], we reported on an initial study of survivability in collisions, with bird strikes as one of the main sources of data. Here is a summary of the preliminary observations:

- Engines. Approximately 50\% of all bird strikes involve the aircraft engine. The number increases to over $75 \%$ for airliners (see, e.g., [Ref. 7]). Patents for jet engine deflectors abound ${ }^{7}$. However, airlines have not adopted any screens for a number of reasons: a screen strong enough to take an impact at aircraft speeds may weigh too much to allow operation; a screen would be a source of turbulence at the intake, causing a potential loss of lift; finally, debris from a broken screen may be a source of secondary damage for the engine.

\footnotetext{
${ }^{6} \mathrm{http}: / / \mathrm{www}$. livescience.com/49701-private-planes-safety.html

${ }^{7}$ http://patents.justia.com/patent/8968437
} 
Engine certification requires designing and planning for operations after bird strikes occur. A "large flocking bird" requirement (2007) stated that the engine must function at a specified performance after ingesting a 5.0 pound bird $^{8}$. It has since been determined that the bird-ingestion design case is not defined by the highest kinetic energy case, but rather by the lowest operating speed, where larger pieces of a bird will cause more damage to the engine core. Despite these requirements, birds have disabled aircraft, pointing to a great deal of uncertainty in the thresholds set in the requirements, as well as the difficulty of including every bird strike scenario. Moreover, existing requirements based on birds would not address the ingestion of a UAS. At high speeds, organic matter has fluid-like behavior, which prevents extrapolation to UAS. Developments in incorporating empirical data into higher-fidelity computational modeling are needed. Computational modeling in [Refs 2,3] compares the effects of UAS ingestion with that of organic matter.

- $\quad$ Agility. Evasive maneuvers may not be an obviously promising direction for avoiding time-critical encounters. A Boeing source ${ }^{9}$ warns pilots: "Avoid or minimize maneuvering at low altitudes to avoid birds". Agile maneuvering to avoid UAS may not be acceptable on a civilian aircraft, even if proven to be structurally and mechanically feasible, due to physiological constraints on passengers.

- $\quad$ Structures. An impact of a bald eagle (average weight 12 pounds) on an airplane climbing at $200 \mathrm{mph}$ would produce the kinetic energy of a half-ton weight dropped from a height of 16 feet. Structural protection may be difficult for even moderate at-rest mass of a colliding object. Military aviation knows examples of non-parasitic armor that has provided great protection against enemy ammunition in combat [Ref. 8]. Although adequate structural enhancements in the form of ruggedized structure, or armor, may appear to be prohibitive in civil aviation in terms of performance and cost effects, consideration of new designs with inclusion of organic ruggedized structures is needed, given the potential severe consequences of collisions. Other structural measures, such as re-arrangement of critical components may be considered.

\section{B. General Comments on Solutions}

The uncertainties and the potentially disastrous outcomes of collisions with non-cooperative UAS (and in particular, with flocks of UAS) suggest that the absolute best solution is prevention. Detecting hazards in time to avoid collisions is infinitely preferable to attempting recovery following a collision. Detect-and-avoid capabilities are in continual development but are not nearly ready for prime time, with perception presenting one of the main difficulties. Interestingly, some of the airspace modernization plans, e.g., the replacement of radar with ADS-B ${ }^{10}$ would make the detection of such obstacles as the flocks of birds more difficult: radars can detect flocks; ADS-B depends on transmission of information.

Given the difficulty of detection and avoidance under the complex conditions of a realistic and dense environment, it is impossible, in principle, to prevent a certain amount of safety degradation in dense autonomous airspace, at least in the foreseeable future. Therefore, an aircraft carrying passengers requires explicit survivability enhancements, to maintain passenger safety.

Adding armor is the least desirable option because of the inevitable loss of performance. However, including the ruggedizing considerations in early stages of design may minimize performance losses. Other survivability measures (maneuvers, control, component and function health monitoring, self-healing, and so on) will require the development of autonomous intelligent systems, to assess and mitigate certain types of collisions, especially those involving several threats at close range.

\section{Potential MDAO Contributions}

Survivability in combat in the military domain has been studied comprehensively (see, e.g., [Refs 8-32]) and offers valuable directions for potential contributions from MDAO. Survivability in the probabilistic framework of [Ref. 8] is the probability of survival and is the complement of killability. Killability is measured along a gradation that includes an inability to complete a mission. In the civilian domain, we can use a more explicit measure - nonsurvivability in collisions, $P_{N S}^{C}$, because the primary mission of the aircraft is to deliver humans on board to the ground safely (and, in addition, not kill anyone on the ground). Not fulfilling the mission of delivering passengers or cargo to a destination, after a collision, still satisfies survivability if everyone survived. Thus,

$$
P_{S}^{C}=1-P_{N S}^{C} \text {. }
$$

\footnotetext{
${ }^{8} \mathrm{https}: / / \mathrm{www}$. skybrary.aero/index.php/Aircraft_Certification_for_Bird_Strike_Risk

${ }_{9}^{9} \mathrm{http} / / /$ www.boeing.com/commercial/aeromagazine/articles/2011_q3/4/

${ }^{10} \mathrm{http} / / /$ airportjournals.com/ads-b-to-replace-expensive-radar-system/
} 
Non-survivability is a function of the aircraft's susceptibility and vulnerability. Susceptibility, $P_{H}^{C}$, is the probability of being hit. Vulnerability, $P_{N S \mid H}^{C}$, is the conditional probability of not surviving upon being hit:

$$
P_{S}^{C}=1-P_{N S}^{C}=1-P_{H}^{C} P_{N S \mid H}^{C} .
$$

Applied to civil aviation, the measure quantifies the chances of survival defined as either not being hit or, if hit, not crashing.

The probabilities are decomposed further, based on specific scenarios and events. The quantification of probabilities in the military domain is done via construction of scenarios, event tree diagrams, simulations, and tests. Improving $P_{S}^{C}$ requires reductions in susceptibility and vulnerability.

\section{Susceptibility}

Susceptibility answers the question: can you get out of harm's way or remove the hazard?

The civilian domain offers susceptibility challenges that differ from those in the military domain, the dissimilarity due to both the aircraft properties and airspace properties. How dense is the airspace? How often does one encounter a non-cooperative aircraft? What are the likely relative velocities and maneuverability properties of both the designed and the likely non-cooperative aircraft? What are the detect/sense/avoid (DSA) capabilities of aircraft under design? Susceptibility can be summarized as a combination of the threat, the aircraft, and the scenario.

In terms of the threat, the civilian domain is easier than the military domain in some respects and harder in others. Encountering a non-cooperative UAS in civilian airspace would likely be a random event, whose probability would depend on the density and attributes of traffic at specific layers, with rare exceptions of malicious participants. Opinions as to the probabilities differ (see e.g., [Ref. 1]), and there are not enough data to formulate a historical model. However various models of the probabilities as a function of density give a good idea of how density impacts chances of collisions with random objects. For instance, [Ref. 33] uses a gas particle model, and it is a reasonable approximation of the worst-case behavior without external regulation of airspace. It is particularly relevant to autonomous and non-cooperative players. One of the examples in [Ref. 33] discusses a case, where the baseline scenario has traffic densities that ensure many hours without 2-body and 3-body interactions. Only a tenfold increase in traffic density yields evasive maneuvers every 20 minutes; with another ten-fold increase resulting in nearly continuous maneuvering, which leads to Brownian motion, rather than getting from point A to point $\mathrm{B}$. The model is a highly idealized simplification of actual traffic, but it shows the trends in probabilities well.

The aircraft contribution to susceptibility is a function of awareness, countermeasures and performance. MDAO does not appear to have an obvious opportunity for contribution in awareness (a function of intelligent systems, both onboard and remote) or in countermeasures. Randomness of the encounters means that, although non-cooperative objects are not as destructive as those in the military domain, their properties are not as known a priori and therefore cannot be predicted or modeled as well as in a combat situation. However, MDAO can play a large role in modeling and design for performance, such as speed and agility of some types of aircraft, given a set of requirements imposed by the likely composition of the airspace. For instance, the density and diversity of vehicles, combined with the range and accuracy of detection may lead to new requirements for aircraft agility.

It is unlikely that airliners will gain great agility. However, if supported by appropriate sensing and automation, an aircraft may not be required to maneuver sharply by a large displacement. If an object is detected in time, even small changes in the path may suffice to weaken collisions with critical sections of the aircraft. Bounds on such actions would be a subject of combined aerodynamics and control study. Flocks of drones would present a more difficult problem, raising the question of adding countermeasures and structural features.

For GA vehicles, the windshield is the most susceptible part of the aircraft: collisions with windshield account for more than 50\% of bird strikes [Ref. 7]. For GA, windshield collisions result in the largest number of fatalities. A clear opportunity for MDAO to contribute here is through design of materials and radical re-design of the cockpit.

\section{Vulnerability}

The measure of vulnerability answers the question of how unlikely an aircraft is to land after being damaged, with the pilots and passengers intact. Because of the random factor in non-cooperative encounters, arguably, vulnerability reduction should be the emphasis of survivability improvement in civil aviation.

In the military domain, vulnerability is addressed via the vulnerability program, which is also practiced in other safety-critical areas. The program has three tasks: identification of critical components and their damage modes; vulnerability assessment; and design for low vulnerability.

The first task depends on the detailed technical description of a specific aircraft. The second stage depends, in addition, on the properties of the threat. The final stage relies on heuristically proposed measures and trade studies to determine relative merits of the proposed solutions. Solutions are based on a number of general principles: 
- Critical component redundancy with separation. For example, multiple engines, control surfaces. NASA's SCEPTOR [Ref. 34] concept with its multiple engines is an example of this principle.

- Critical component location and separation to prevent cascading damage.

- Passive damage suppression via fault tolerant design and such technologies as high-strength and selfhealing materials.

- Active damage suppression via technologies that incorporate sensors, e.g., fire detection and suppression.

- Critical component shielding by other components or additional structure. Model development is needed here to avoid parasitic shielding.

- Critical component elimination. Replacing pilots with automation comes under this category.

The principles of vulnerability reduction apply to civilian aircraft and to other safety-critical areas; and have been used in civil aviation to reduce vulnerability with respect to mechanical failure. However, the approach presents opportunities for improvement with MDAO techniques, especially in the design of new aircraft, as follows.

\section{Vulnerability as Objective/Constraint}

The probabilistic evaluation of vulnerability in the military domain appears to be based on a great deal of detailed design having been accomplished; vulnerability is an outcome of applying heuristic principles during design and an a posteriori evaluation; followed by corrections, which might require re-design. The trend in design of civilian aircraft is to increase discipline fidelity at earlier stages of design, to improve the quality of design and shorten the design cycle time. A purely probabilistic, post factum evaluation of vulnerability cannot be easily used as an objective function or a constraint in an MDAO formulation. Developing a computational model of vulnerability for use in MDAO formulation would be necessary.

\section{Uncertainty Quantification}

Given the need for a higher degree of survival assurance of each civilian aircraft carrying passengers, a great deal of accuracy would be needed for uncertainty quantification and propagation in the evaluation of vulnerability. Uncertainty quantification in the military domain is related to the characteristics of known weapons. Developing uncertainty quantification of impact for possible collision sources in civilian domain is needed. A recent study [Ref. 35] investigated UAS impact damage using the FAA penetration equation $V_{50}=\sqrt{\frac{2 L C_{S} t^{2}}{m \cos ^{2} \theta}}$, where $V_{50}$ is the ballistic limit, i.e., the velocity required to make a hole in a sheet of metal. Here $m$ is the mass of the projectile, $\theta$ is the angle of the impact, $C_{S}$ is a material property constant, $L$ is the perimeter of the presented area of the projectile, and $t$ is the thickness of the metal sheet. The analysis indicated that engine ingestion in an airliner, the likeliest event with probable loss of engine, is unlikely to be catastrophic. (However, the analysis was done for a single UAS; not a flock of UAS.) The analysis further predicted penetration of aluminum airframe for velocities 200 knots and above, regardless of UAV size and penetration predicted for fixed-wing UAV at low velocities. For GA aircraft, penetration was predicted likely at cruise speeds, regardless of UAV size; and likely for large UAV during approach. Windshield penetration results were highly uncertain as they depend on material properties and angle of the windscreen. These results are informative of the trends, but a significant increase in model fidelity is needed to reduce analysis uncertainty.

\section{Dimensionality}

Given thousands of components on an aircraft, the approach to identifying critical components, damage modes and potential mitigation via discrete enumeration is likely to give rise to problems of very large dimension, whereas reducing the design space for tractability may cause missed salient failure conditions or interesting designs. Appropriate problem decomposition and aggregation is a fruitful area of investigation for MDAO.

\section{Reliability, Robustness and Resilience}

Traditional reliability-based design and robust design address the majority of the issues that may arise as a result of damage inflicted by a collision. Whether the loss of all flight controls results from a catastrophic tail-mounted engine explosion, as it did on United Airlines Flight 232 DC-10 in 1989 in Sioux City, Iowa ${ }^{11}$ or from a prospective collision with a flock of UAS, the design measures of mitigation might be similar. However, different event probabilities may dictate different approaches to reliable designs. On the one hand, the three independent hydraulic systems on board of a DC-10 conformed to the principle of component redundancy. On the other, placed together,

${ }^{11}$ https://en.wikipedia.org/wiki/United_Airlines_Flight_232 
they were at odds with the principle of critical component separation. Overall, the probability estimation of the event was based on an incorrect assumption that failures of the three hydraulic systems would be independent events. Given increased probabilities of collision, critical component redundancy and separation may become the main principle, which would call for a rigorous approach to uncertainty quantification and a formal optimization-based approach to component placement.

In the environment of dense and diverse autonomy, reliability (operation in worst-case scenarios) has to be considered in close coordination with other related concepts of robustness (insensitivity to normal variations in operational parameters) and resilience (the ability to return to operation following a disturbance, even if only to reach a satisfactory final state).

Whether vulnerability to collisions is viewed as a subset of the discipline of reliability or vice-versa, revisiting reliability, robustness and resilience from the perspective of vulnerability and survivability becomes especially important in the new environment because of the potential proliferation of new small aircraft and UAS designs that would not be subject to the careful engineering of airliners.

The probabilistic definition of survivability, including susceptibility and vulnerability, should include reliability of the design to both collision-related damage and non-collision related failures.

\section{Survivability on the Ground}

Increased UAS traffic and the projected appearance of air taxis and private air vehicles, if realized, will increase risk to people on the ground, animals, and property in highly populated areas. The risk could be mitigated by restricting air traffic to specific air corridors. However, this measure would be at odds with the objectives of ondemand transportation (ODM), ultimately aimed at door-to-door delivery of cargo and people.

It stands to reason that all measures aimed at ensuring the four elements necessary for flight - lift, thrust, control, and structural integrity - should be taken; e.g., intelligent systems performing continual monitoring of the health of the aircraft through sensors, improvements in maintenance, machine-pilot teaming to prevent pilot error (and eventually replacing the pilot with autonomous intelligent systems where appropriate), intelligent technologies in general (e.g., perception, real-time safety-critical decision-making for collision detection and avoidance, intelligent control); and design improvements (e.g., self-healing materials, propulsion control, resilient structures). Some of these measures are available now but safety and cost are trade-off quantities. Other measures have not reached their potential yet. Regardless, increased density implies increased chances of crashes.

What happens if an aircraft is damaged would depend on the type of the aircraft. In case of a UAS, one promising approach is exemplified in the Safe2Ditch ${ }^{12}$ technology, which helps a malfunctioning UAS select the best and nearest emergency landing site and land. The system is based on continual monitoring of the UAS components, to detect imminent failure and control the system to maintain flight till the UAS reaches a landing site recommended by a database of safe landing sites and associated safe times.

If a UAS is unable to land harmlessly, other approaches can be considered, depending on the attributes of the UAS, including the nature of its cargo. For instance, the use of a parachute or self-destruction may be viable options for an unmanned UAS unable to accomplish a safe or gentle landing.

Design for landing harmlessly is a promising area for MDAO techniques in the case of aircraft carrying humans, because such techniques may involve a re-design of the aircraft. One obvious approach that has had significant success is a ballistic recovery system ${ }^{13}$. As of mid-August 2016, the Cirrus Airframe Parachute System (CAPS) was successfully deployed 69 times out of 83 activations, with 142 survivors and one fatality. No fatalities, unsuccessful deployments or anomalies have occurred when the parachute was deployed within the certified speed and altitude parameters $^{14}$. CAPS focuses on ultralight vehicles. Larger vehicles may be amenable to parachuting if separable modules are equipped with ballistic recovery systems.

Other design considerations ${ }^{15}$ for reducing the chances of crashes usually meet with cost limitations. MDAO could contribute by incorporating comprehensive cost modeling in the analysis of new configurations resistant to crashing and amenable to harmless landing.

\footnotetext{
${ }^{12}$ http://newatlas.com/nasa-safe2ditch-dronesafety/49489/?utm_source=Gizmag+Subscribers\&utm_campaign=d2ec2a7708-UA-22353604\&utm_medium $=$ email\&utm_term $=0$ 65b67362bd-d2ec2a7708-91183197

${ }^{13} \mathrm{https}$ ://en.wikipedia.org/wiki/Ballistic_Recovery_Systems

$14 \mathrm{https}: / /$ www.cirruspilots.org/copa/safety_programs/w/safety_pages/723.cirrus-caps-history.aspx

${ }^{15} \mathrm{http}: / / \mathrm{www}$. economist.com/news/science-and-technology/21660255-towards-crash-proof-aeroplane-quit-stalling
} 
In any case, the probabilistic definition of survivability, including susceptibility and vulnerability, should include considerations of potential aircraft lethality to people on the ground. Thus, the total measure of vulnerability would be the sum of aircraft vulnerability to mechanical failure, vulnerability in collisions and vulnerability of people on the ground should the aircraft fall from the sky.

\section{Total Survivability as a Challenge MDAO Problem}

Methods for increasing survivability in the military domain are a combination of rules, best practices, and probabilistic computations. Numerical values of survivability and its contributing factors - susceptibility and vulnerability - can be computed as probabilities of complex sequences of events; and improved by following the vulnerability reduction heuristics. The assessment of survivability, however, depends on the knowledge of a vehicle's detailed design. Thus significant design effort is expended before survivability can be evaluated.

Arguably, two of the main benefits of MDAO are the transition from discrete heuristic point designs to rigorous, optimization-based formulations, with early communications among the disciplines; and the shortening of the design cycle via prevention of premature detailed designs based on insufficient information. However, the application of MDAO techniques requires adequate modeling of the contributing disciplines and the ability to incorporate them in optimization formulations, including objective and constraints expressed as functions of design variables. In this respect, survivability presents a complex technical problem.

Survivability is affected by contributions from all design disciplines: structures, agility, materials, propulsion, control, etc. all influence susceptibility and vulnerability. However, the connection between the traditional vehicle design variables and the measures of survivability is implicit and inferred.

In a typical operational scenario, the sensory systems of the aircraft must continually monitor the external environment and internal state of the aircraft to inform the intelligent autonomous perception and decision making system, which, in turn, issues commands to the aircraft, to avoid a collision, weaken it, or recover sufficiently to deliver the passengers to the ground safely. The traditional disciplines are responsible for the actions dictated by the intelligent system to be physically implementable. In order to achieve this, survivability must be directly connected, through variables, objectives and constraints, with all relevant disciplines.

Given the state of the art in contributing disciplines and MDAO, including survivability into MDAO on par with traditional computational disciplines presents a difficult challenge: for instance, adequate collision modeling would be computationally intractable and the required interdisciplinary interfaces have not been developed. Inclusion of survivability necessitates developments in computational modeling, manufacturing, materials, and MDAO problem synthesis, among other technologies.

In this sense, survivability appears similar to the discipline of cost. Cost can be evaluated as an aggregate of parts, but it is difficult to include cost as an objective function in MDAO, on par with objectives from the physicsbased disciplines, even though this goal has been among the desiderata of MDAO for a long time. However, given that survivability is related to reliability, there is hope that its formalization in terms of explicit design variables and associated objectives and constraints is not an insurmountable obstacle. Incorporating both survivability and cost into design formulations would be an even better approach, so that the trade-off between survivability and cost would be made explicit.

\section{Concluding Remarks}

Although the actual realization of the future airspace is uncertain, there are clear trends in the direction of increased density of traffic, and diversity and autonomy of vehicles. If the trends continue, complexity of airspace, especially over densely populated areas, will increase the chances of crashes due to mechanic and electronics failures, errors in machine decisions, human error, and mid-air collisions.

While many sources of risk reduction rely on intelligent technologies, such as DSA, designing aircraft that ensures unprecedented reliability and resilience in the face of hazards, as well as graceful degradation of the systems, to prevent injuries, fatalities, and damage on the ground will play a major role in safe aviation. Developing adequate and actionable models of survivability is a challenge for MDAO. Of particular interest is a conversion of the vulnerability reduction principles to formalizations of design optimization. The difference between traditional MDAO and MDAO with survivability as an objective is the need to include airspace considerations in vehicle design explicitly. 


\section{Acknowledgments}

The author would like to thank the Incubation element of the NASA Convergent Aerospace Solutions (CAS) Program for supporting this work during the period 10/01/2015-09/30/2016.

\section{References}

[1] Weibel, Roland E. and Hansman, John R.; Safety Considerations for Operation of Unmanned Aerial Vehicles in the National Airspace System; Report No. ICAT-2005-1, MIT, March 2005

[2] Song, Yangkun, Horton, Brandon; Bayandor, Javid; Investigation of UAS Ingestion into High-Bypass Engines, Part I: Bird vs. Drone; 58th AIAA/ASCE/AHS/ASC Structures, Structural Dynamics, and Materials Conference (doi: 10.2514/6.20170186)

[3] Schroeder, Kevin; Song, Yangkun, Horton, Brandon; Bayandor, Javid; Investigation of UAS Ingestion into High-Bypass Engines, Part II: Drone Parametric Study; 58th AIAA/ASCE/AHS/ASC Structures, Structural Dynamics, and Materials Conference, AIAA SciTech Forum, (AIAA 2017-0187), http://dx.doi.org/10.2514/6.2017-0187

[4] Introduction to TCAS II, Version 7.1; US Department of Transportation, Federal Aviation Administration, 28 Feb. 2011

[5] Kochenderfer, Mykel J.; Holland, Jessica E.; Chryssanthacopoulos; Next-Generation Airborne Collision Avoidance System; Lincoln Laboratory Journal, Vol. 10, Num. 1, 2012

[6] Alexandrov, N.; Ozoroski T.; Design for Survivability: An Approach to Assured Autonomy, AIAA 2016-4374

[7] Thorpe, J.; 100 Years of Fatalities and Destroyed Civil Aircraft due to Bird Strikes; 30th Meeting of the International Bird Strike Conference; 25-29 June 2012, Stavanger, Norway

[8] R. E. Ball, The Fundamentals of Aircraft Combat Survivability Analysis and Design, Second Edition, AIAA Education Series, 2003

[9] L. M. Nicolai, G. E. Carichner, Fundamentals of Aircraft and Airship Design, AIAA Education Series, 2010

[10] D. B. Atkinson, P. Blatt, L. Mahood, D. W. Voyls, Design of Fighter Aircraft for Combat Survivability. Sae Transactions. 78, 159-\& (1969).

[11] J. O. Berger, Wald, Abraham Work on Aircraft Survivability - Comment. Journal of the American Statistical Association. 79, 267-269 (1984).

[12] D. Brown, C. Miller, M. Schumacher, K. Werner, S. T. Crino, Improving Special Operations Aircraft Survivability through Better Ballistic Protection Systems , 2009), pp. 158.

[13] J. Clements, R. Robinson, L. Bunt, J. Robinson, Missile Airframe Simulation Testbed - MANPADS (MAST-M) for Test and Evaluation of Aircraft Survivability Equipment. Technologies for Synthetic Environments: Hardware-in-the-Loop Xvi. 8015, 80150A (2011).

[14] S. C. Colwell, A Cost-Effective Method to Determine Ground Survivability of Strategic Aircraft. Oper. Res. S 14, B203$\&(1966)$.

[15] A. H. Cooper, Survivability of Military Vstol Transport Aircraft. Sae Transactions. 78, 159-\& (1969).

[16] L. Jia, Z. Tong, C. Wang, S. Li, Aircraft Combat Survivability Calculation Based on Combination Weighting and Multiattribute Intelligent Grey Target Decision Model. Mathematical Problems in Engineering. 8934749 (2016).

[17] J. Kang et al., Aircraft Survivability and Sensors Alignment Techniques. Journal of the Korean Society for Aeronautical Science and Flight Operation. 16, 29-36 (2008).

[18] F. Leary, Tactical Aircraft Survivability. Space Aeronautics. 47, 68-\& (1967).

[19] K. Lee, et al. Aircraft Combat Survivability Analysis based on the Random Variable Weighted Score Algorithm. Journal of the Korean Society Aeronautical and Space Sciences. 41, 883-890 (2013). .

[20] M. Mangel, F. J. Samaniego, A. Wald, Work on Aircraft Survivability. Journal of the American Statistical Association. 79, 259-267 (1984).

[21] M. Mangel, F. J. Samaniego, A. Wald, Work on Aircraft Survivability - Rejoinder. Journal of the American Statistical Association. 79, 270-271 (1984).

[22] G. I. Nesterenko, Service life and survivability of aircraft structures. Materials Science. 32, 166-178 (1996).

[23] S. Noh, C. Choi, Predicting the Operational Effectiveness of Aircraft Survivability Equipment Suite. International Journal of Engineering and Technology. 4, 372 (2012).

[24] V. S. Safronov, S. E. Zaytsev, M. Y. Kalajgin, Estimation Of Survivability Of The Aircraft Wing Design Taking Into Account Damage Accumulation. Polet.Obshcherossiiskii Nauchno-Tekhnicheskii Zhurnal., $46-54$ (2012).

[25] V. S. Safronov, S. E. Zaytsev, M. Y. Kaljagin, Optimal Design Console Wing Aircraft With The Requirement Of Survivability Of The State Aviation. Polet.Obshcherossiiskii Nauchno-Tekhnicheskii Zhurnal., 40-50 (2013).

[26] V. S. Safronov, Accounting Requirements for the Design Survivability of Shell Structures Aircraft. Aviakosmicheskoe Priborostroenie., 17-25 (2015).

[27] V. S. Safronov, Accounting Requirements for the Design Survivability of Flat Panel Aircraft. Aviakosmicheskoe Priborostroenie., 55-60 (2015).

[28] R. Soannini, P. Froment, Survivability Increase of Military Transport Aircraft. Nouvelle Revue Aeronautique Astronautique., 44-52 (1995).

[29] L. Throndson, Combat Survivability with Advanced Aircraft Propulsion Development. J. Aircr. 19, 915-920 (1982). 
[30] J. M. Vice, Survivability Vulnerability Information Analysis Center (Surviac) - a Tool for the Aircraft Survivability Community. J. Aircr. 24, 511-515 (1987).

[31] H. Wang, S. Li, N. Chen, Simulation and Analysis of Aircraft Survivability Model the Impacted by Rain. Fire Control \& Command Control. 8, 020 (2012).

[32] X. Wang, B. Song, Y. Hu, Analytic Model for Aircraft Survivability Assessment of a One-on-One Engagement. J. Aircr. 46, 223-229 (2009).

[33] Alexander, Ben; Aircraft Density and Midair Collision, Proceedings of the IEEE, Vol 58, No. 3, March 1970

[34] Moore, Mark; Clarke, Sean; Convergent Aeronautics Solutions Project - SCEPTOR - Scalable Convergent Electric Propulsion Technology and Operations Research; DFRC-E-DAA-TN37115

[35] Radi, Alexander; Potential damage assessment of a mid-air collision with a small UAV, Civil Aviation Safety Authority, Monash University, 12/6/2013 